\title{
Impact of Physical Activity and Lifestyle in Pre- infection on the Susceptibility and Prognosis of Infects With COVID-19
}

\section{Baozhen Huang}

2nd Medical Center of Chinese PLA General Hospital

\section{Shixi Zhang}

Shangqiu Municipal Hospital

Jiuyu Gong

the Hubei Armed Police Crops Hospital

\section{Yanlin Niu}

Beijing Center for Disease Prevention and Control

\section{Fengjuan Zhang}

The Affiliated Hospital of Qingdao University

\section{Lin Jiang}

the Hubei Armed Police Crops Hospital

\section{Mingming Wang}

Shangqiu Municipal Hospital

Jing Cui

Shangqiu Municipal Hospital

\section{Weixin $\mathrm{Li}$}

First People's Hospital of Jiangxia District

Cheng Jin

2nd Medical Center of Chinese PLA General Hospital

\section{Pengtao Bao}

8th Medical Center of Chinese PLA General Hospital

Diangeng Li ( $\square$ lidiangeng@126.com )

2nd Medical Center of Chinese PLA General Hospital https://orcid.org/0000-0002-8698-4746

\section{Research}

Keywords: COVID-19, Physical Activity, Length of Stay, Sleep, Exercise, Asymptomatic

Posted Date: July 23rd, 2020

DOI: https://doi.org/10.21203/rs.3.rs-46730/v1 
License: (c) (i) This work is licensed under a Creative Commons Attribution 4.0 International License. Read Full License 


\section{Abstract \\ Background}

There are very few studies focusing on the relationship between COVID-19 and pre-infection lifestyle. In the absence of effective vaccines and special-effect medicines, it is very meaningful to actively respond to the disease pandemic by improving lifestyle habits.

\section{Methods}

This is a multicenter, retrospective cohort study enrolled 431 adult people including 228 normal people and 203 confirmed infects in Wubei, Henan and Shandong Provinces. Questionnaires were used to collect information on physical activity and lifestyle by competent doctors. The univariate logistic regression models and multiple regression models were used in risk factor analysis. Kruskal-Wallis $\mathrm{H}$ test were used to test the correlation.

\section{Results}

Lifestyle habits including exercise, smoking, sleep and physical activity can significantly affect the probability of getting COVID-19 $(P<0.05)$. The MET (Metabolic Equivalent) intensity classification and sleep status are found to be the potential influencing factors of prognosis in both all infects and symptomatic patients. In all infects, taking the high MET intensity level as a reference, inpatient days would increase by 1.812 times ( $95 \% \mathrm{Cl}$ : $0.887-3.701)$ with no significance when the level is moderate ( $P$ $>0.05)$ and significantly increase by 6.674 times $(95 \% \mathrm{Cl}: 1.613-27.613)$ when the level is low $(P<0.05)$. Kruskal-Wallis $H$ test results showed moderate activity MET*min promoted shorter hospital stay $(P<0.05)$ mainly.

\section{Conclusions}

Sleep status and physical activity influenced the susceptibility and prognosis of COVID-19. Lack of sleep and low MET intensity level may prolong the hospital stay, which means a relatively slow recovery. This encourages the public to have moderate physical activity and adequate sleep to respond to the COVID-19 pandemic actively.

\section{Introduction}

Since November 2019, the rapid outbreak of coronavirus disease 2019 (COVID-19), which arose from severe acute respiratory syndrome coronavirus 2 (SARS-CoV-2) infection, has recently become a public health emergency of international concern ${ }^{1}$.There have been $6,750,521$ laboratory-confirmed cases 
and395,779 deaths globally as of 7 June, $2020^{2}$.Several recent retrospective studies have suggested that age and underlying diseases, including hypertension, diabetes,respiratory and cardiovascular disease may be risk factors for adverse outcomes in COVID-19 ${ }^{3-5}$.Some studies demonstrated that infection and severity of COVID-19 are associated with smoking while there is no very conclusive conclusion ${ }^{6-8}$. There are very fewstudies focusing on the relationship between COVID-19 andpre-infection lifestyle. In the absence of effective vaccines and special-effect medicines, it is very meaningful to actively respond to the disease pandemic by improving lifestyle habits. Furthermore, most of the existing studies which aimed to explore therisk factors for disease only included symptomatic patients ${ }^{4,9,10}$. However, the study of positive COVID-19 infections onboard the Princess Cruises ship showed that the ratio of asymptomatic infect is $17.9 \%(95 \% \mathrm{Cl}: 15.5 \%-20.2 \%)^{11}$.Studies on risk factors should include both symptomatic and asymptomatic patients. Objective of this study was to find out the relationship between prognosis of COVID-19 and pre-infection lifestyle including exercise, sleep habit, physical activity and other personal habits. Besides through a more comprehensive survey, we investigate the infected and uninfected people in the same area, thus we can explore the susceptibility factors from the perspective of lifestyles.

\section{Materials And Methods}

\section{Participants and data collection}

There are 4 clinical centers in 3 provinces including Hubei, Henan and Shandong provinces had been selected in this study. Infected adults who discharged between 10 Feb 2020 and 28 March 2020, including both symptomatic and asymptomatic infections, were admitted. The adult normal people of the corresponding period were enrolled as the control group. Criteria for infects inclusion were as follows: 1) a history of COVID-19 infection confirmed by high-throughput sequencing or real-time reversetranscription polymerase-chain-reaction (RT-PCR) assay findings for positive nasal and pharyngeal swab specimens ${ }^{12}$;2) infections of Chinese nationalityand above 18 years old. People with severe communication problems (e.g., deafness, dementia, mental retardation)were excluded. With the help of doctors in charge, participants wouldcomplete a questionnaire focused on physical activity and lifestyle (Supplementary material 1) willingly via telephone follow up.The detailed information regarding exercise habits, physical activity and sleep status of the participants before the infection were investigated in the questionnaire. All calls were recorded and saved anonymously with the oral informed consent of the participant.The personal information (including age, gender, weight, height) and the clinical data (including clinical symptoms and signs, treatment, comorbidities, and laboratory findings from admission to discharge) were reviewed and extracted by doctors in charge of the patients.Manifestations on computed tomography (CT) were summarized by integrating the documentation or description in medical charts and, if available, a further review will be conducted by the medical staff. The records of Brag's score(Supplementary material 2)that can reflect the patient's breathing were filled in by the patients at the time of admission and discharge ${ }^{13}$.

\section{Definition of the variables}


The inpatient days of patient with COVID-19 is considered as the primary outcome in this study, which is associated with the speed of virus testing from positive to negative according to discharge standard ${ }^{14}$. The length of hospital stay is divided into three levels, 0-9 days,10-19days, and over 19days according to experience in designated treatment hospitals. The definitions of smoking history and underlying disease history are consistent with the previous research ${ }^{15}$. BMI classification is categorized as normal (18.523.9) and overweight (>24) according to cut off points in Chinese definition ${ }^{16}$. Having exercise habit means taking exercise activities that meet the exercise standard every week in the daily activities before a diagnosis of COVID-19, including aerobic, anaerobic and flexibility training, but excluding ordinary walking and fishing intensity activity, which is defined by the National Heart Association ${ }^{17}$. Having regular exercise is defined as do exercise activities as above at amore than or equal to three times a week and no less than 30 minutes each time. International Physical Activity Questionnaire (IPAQ) -Short Form is used to identify the physical activity, sleep status and sedentary behavior of the COVID-19 infections ${ }^{18}$. The intensity of physical activitiesare described using Metabolic Equivalents (METs). To calculate the MET minutes a week, the MET value given (walking = 3.3, moderate activity $=4$, vigorous activity $=8$ ) is multiplied by the minutes in the activity and again by the number of days that activity. The sum of MET minutes a week for walking, moderate activity and vigorous activity is calculated as the total MET minutes of physical activity a week, and the sum of that for the latter twois calculated as moderateintensity MET minutes of physical activity a week.Activity bouts in each category of greater than 3 hours are truncated. The level of physical activity intensity is divided into high, medium and low, according to the standards set by the IPAQ committee ${ }^{19}$.Sedentarybehavior is defined as usually sitting for 9 hours or more per day on working days ${ }^{20}$. In addition, the average sleep time in hours per day was converted into a categorical variablelabelled with recommended, may be appropriate and lack of sleep, according to NSF (National Sleep Foundation) guidelines ${ }^{21}$.

\section{Statistics}

Quantitative data were presented as means with standard deviations. Categorical data were presented as percentages of the population in groups. In the comparison between groups, the independent sample ttest was used for the continuous variables. The chi-square test was used for categorical variables and was adjusted when needed. Simple univariable logistic regressions and binary logistic regression model were used to explore the potential susceptibility factors.Simple univariable logistic regressionsand the ordinal logit regression were used to explore the influencing factors in pre-infection lifestyle of prognosis of patients with COVID-19. All variables with $P$ values $<0.10$ in univariate analyses and other variables of special interest were included in the multiple regression model. Odds ratios (OR) and 95\% confidence intervals $(\mathrm{Cl})$ were listed. For multivariable analysis, $P$-value $<0.05$ was considered statistically significant. Kolmogorov-Smirnov test was used to conduct the normality test for variables and Kruskal-Wallis $H$ test was used to further examine the difference in groups when the data did not coincide with the normal distribution. Data were analyzed using SAS 9.2 (SAS Inc., Cary, North Carolina, USA).

\section{Results}


There were 431 people been selected in this study in total. 228 adult normal people in these areas were invited to do a telephone interview, and $82.5 \%$ (188/228) of them answer the doctor's telephone questionnaire on lifestyle. A total of 203 infected adults were admitted to the study cohort. $80.7 \%$ $(164 / 203)$ of them completely answered the questionnaire via telephone follow up. There were 23 asymptomatic infections and 141 symptomatic infections. In group of asymptomatic infections, $56.52 \%$ $(13 / 23)$ of patients are male and the largest age group is $20-39$ which accounted for $52.17 \%$. While in group of symptomatic infections, $48.94 \%(69 / 141)$ of patients are male and the largest age group is 40 59 which accounted for $45.39 \%$.

\section{Characteristics of COVID-19 infects}

According to the report of Diamond Princess and the retrospect of the transmission of asymptomatic infections in Anyang, China, asymptomatic infections has the ability to spread and the proportion of them was relatively high ${ }^{12,22}$. In this cohort, $14.02 \%(23 / 164)$ people were asymptomatic, and $47.8 \%(11 / 23)$ had no signs of lung infection during hospitalization (Normal lung CT). It is not easy to identify asymptomatic infects that may lead to the presence of an invisible transmission chain. From Supplementary Table 1, there were no significant differences in gender, age, BMI classification, underlying disease between asymptomatic and symptomatic infections ( $P>0.05$ ). Smoke history showed significant difference between groups and proportion of people with smoke history in asymptomatic group was higher than that in symptomatic group ( $P=0.008)$. Regarding the clinical symptoms, the asymptomatic group had less dyspnea on admission ( $P<0.001)$, higher counts of white blood cells $(P=0.012)$ and a lower C-reactive protein level $(P<0.001)$ that reflected inflammation. In terms of lifestyle, there was no significant difference in exercise habits, regular exercise, total MET*min, walking MET*min, vigorous and moderate MET*min, MET intensity classification, sedentary behavior, and the average sleep time per day $(P>0.05)$. The difference between the two groups were found in vigorous-level physical activity MET*min $(P=0.034)$ and moderate-level physical activity activity*min $(P=0.001)$. Asymptomatic infected people have more vigorous MET* $\min (1794.78 \pm 2920.51$ to $279.77 \pm 970.20)$ and relatively few moderate MET*min $(957.39 \pm 1043.08$ to $2322.34 \pm 1807.53)$ compared with symptomatic patients.

\section{Lifestyle habits may affect the probability of getting COVID-19}

We investigated the lifestyles of the patient group and the non-patient group in the area where the patient group is located. And we tried to find whether these lifestyle-related factors affected the probability of disease. Gender, age, BMI, smoke history, underlying disease, exercise habit, sedentary status, physical activity and sleep status were incorporated into a univariate regression model to explore the influencing factors of getting infection of COVID-19. The details are showed in Supplementary Table 2. The variables whose univariate analyses yielded $p$-values $<0.10$ were included in the multivariable logistic regression model in Table 1. History of smoking ( $P=0.004)$, having underlying disease $(P<0.001)$, irregular exercise $(P=0.015)$ and sedentary population $(P=0.003)$ are independent risk factors for getting the disease. The lifestyle of irregular exercise can increase the risk of illness by 2.558 times ( $95 \% \mathrm{Cl}: 1.202-5.445)$. And the lifestyle of sedentary population can increase the risk of illness by 36.054 times ( $95 \% \mathrm{Cl}$ : $3.444-377.443)$. 
Among the non-sick people, there were few sedentary people. Longer sleep time significantly protected people from disease $(P<0.001)$. However, according to NSF's recommendations for people of different ages, proper sleep does not seem to reflect the protection of health. We speculate that this is because the definition of "probably appropriate" in Sleep Status variable includes two types of long sleep time and short sleep time, which are divided according to different ages. This classification is not appropriate in the COVID-19 susceptibility study. For physical activity, the moderate-intensity physical activity was a protective factor against COVID-19 compared to high-intensity physical activity $(P=0.002)$. Relative lowlevel physical activity was even slightly better than high-intensity physical activity ( $P=0.002$, OR 0.078 (95\%Cl: 0.028-0.218)). Too much physical activity may reduce immunity and be susceptible to viruses.

\section{Physical activity intensity and sleep status can significantly affect the hospital stay length of all COVID- 19 infects.}

Result of univariate logistic regression (Supplementary Table 3) showed that smoke history, MET intensity classification, and sleep status were statistically significant $(P<0.10)$. These variables and those with clinically significant variables in the study were then employed into the ordinal logit model. Result of the ordinal logit regression (Table 2) showed that MET intensity classification and sleep status had significant effects on the hospital stay. Taking the high MET intensity level as a reference, hospital stay would increase by 1.812 times ( $95 \% \mathrm{Cl}$ : 0.887-3.701) with no significance when the level is moderate ( $P>0.05)$ and significantly increase by 6.674 times (95\% Cl: 1.613-27.613) when the level is low $(P=0.009)$. As for the sleep status, compared with the recommended, the risk of prolong hospital stay would increase by 2.287 times (95\% Cl: $0.951-5.502)$ with no statistically significant $(P>0.05)$ if the sleep status was may be appropriate. While lack of sleep can significantly increase the risk of 5.525 times (95\% Cl: 1.284-23.770, $\mathrm{P}=0.022)$.

\section{Moderate physical activity plays an important role in reducing the hospital stay length of all COVID-19 infects.}

The classification of MET intensity is mainly based on the weekly frequency in addition to each MET * min, which is a comprehensive evaluation result ${ }^{19}$. To look more closely at the impact of each physical activity category, the comparisons of vigorous activity MET*min, moderate activity MET*min, walking MET*min, and the sum of vigorous with moderate activity MET*min in groups of hospital stays were conducted using Kruskal-Wallis $\mathrm{H}$ test. The results in Table 3 showed moderate activity MET*min $(P=0.015)$ and the sum of vigorous activity MET*min with moderate activity MET*min $(P=0.025)$ had a significant influence on the length of hospital stay, which suggested that those who have a moderate physical activity level before being infected may recover faster from COVID-19 than others.

\section{Physical activity intensity and sleep status can significantly affect the hospital stay length of symptomatic patients.}

In the previous study of the entire cohort, we found that good sleep and moderate physical activity can affect the length of hospital stay. Since some studies have shown that the clinical manifestations and 
epidemiological characteristics of asymptomatic infects are elusive ${ }^{23,24}$, and our cohort contains asymptomatic patients, we re-select symptomatic patients to conduct the analysis. The results of univariate logistic regression of each independent variable for symptomatic patients are in Supplementary Table 4. Based on the results of the ordinary logit model for symptomatic patients in Supplementary Table 5, the MET intensity classification and sleep status were also statistically significant $(P<0.05)$. The inpatient days increased with the reduced physical activity intensity rating $(P<0.05)$, which could increase by 2.289 times (95\% Cl: $1.051-4.983)$ and 11.370 times (95\% Cl: 1.969 65.644) for the moderate and low level of the MET intensity, respectively. Lack of sleep remained a significant risk factor ( $P<0.05)$, in which the OR was 4.816 (95\% Cl: 1.108-20.937) compared with the recommended sleep status. Supplementary Table 6 shows the results of Kruskal-Wallis $\mathrm{H}$ test of each physical activity intensity in groups of hospital stay in symptomatic patients. The same as the whole cohort, moderate activity MET*min $(P=0.002)$ and the sum of vigorous with moderate activity MET*min were significant $(P=0.009)$.

\section{Discussions}

Before the outbreak of the pandemic, due to the absence of the isolation policy and the advent of the traditional Chinese New Year holiday, the enormous population flowing brought uncertainty to the spread of SARS-COV-2. Based on our research on whether people in the same area got COVID-19, smoking history, having underlying disease, irregular exercise and sedentary population are independent risk factors for the disease. Longer sleep time and taking moderate-intensity physical activity were protective factors against COVID-19. Many studies showing that lifestyle can affect flu susceptibility, poor lifestyle habits may increase the likelihood of getting sick by affecting immunity and biological clock ${ }^{25,26}$. As a complex living body, human life style details seem to affect the susceptibility to diseases to some extent.

Though there are plenty of studies focusing on risk factors of the mortality and progress of COVID-19 10 , 15,27 . The influence of lifestyle, especially physical activity and exercise habit, are still unclear. The substantial data were supporting an inverse relationship between the amount of habitual physical activity performed and a variety of adverse health outcomes throughout the lifespan ${ }^{28}$. Thus, we further analyzed the relationship between lifestyle and hospital stay length of the patients.

The length of hospitalization and the mortality rate are considered to be two essential indicators in clinical trials of drugs ${ }^{29,30}$. Since the patients who can discharge from the hospital must achieve repeated negative SARS-CoV-2 RNA tests (more than one day apart) and the significant symptom improvement, the length of hospital stay reflects the speed of virus clearance and recovery. The longer hospital stay and treatment process not only bring pain and inconvenience to patients, but also increase their financial burden. At the same time, in areas where the number of beds is saturated, the occupied beds prevent the hospital from accepting more new patients and cause delays in the treatment of new COVID-19 patients ${ }^{31}$. Both in all COVID-19 infects and symptomatic patients, we got the same results. MET intensity classification and sleep status had significant effects on the hospital stay. Lack of sleep 
can significantly increase the risk of longer inpatient days. Although the length of hospitalization increases as the intensity of physical activity decreases, by category, medium physical activity MET*min seems to play a decisive role.

Suitable physical activity is believed to be beneficial to cardiovascular health and can reduce all-cause

mortality ${ }^{32,33}$. Sedentary women and overweight children can benefit from increased physical activity 34,35 . But scientist also demonstrated too much high-intensity physical activity has a potential risk of causing death ${ }^{32}$. Especially the short-term, high-intensity exercise can lead to a significant and prolonged dysfunction of the mitochondrial energy status of peripheral blood leucocytes with an increased propensity for apoptosis and raised pro-inflammatory mediators ${ }^{36}$. Meanwhile, intense exercise causes immunosuppression, while moderate-intensity exercise improves immune function and potentially reduces the risk and severity of the respiratory viral infection. Moderate exercise-induced increases in stress hormones reduce excessive local inflammation and skew the immune response away from a Th1 and towards a Th2 phenotype, thus improving outcomes following respiratory viral infection ${ }^{37}$. According to our research results, moderate-intensity physical activity is most conducive to shortening COVID-19 hospitalization days, which is consistent with international consensus on physical activity.

The limitation of this study is limited sample size and the credibility of the subjective questionnaire. However, we included the infected people in the four medical centers in three provinces of China to increase the representative of the sample as far as possible. We also asked the doctor in charge to do the questionnaire by telephone as much as possible to ensure data accuracy. Moreover, we began to do more large-scale and in-depth research to track and investigate the impact of exercise habits and physical activity on the long-term rehabilitation of COVID-19 to give the most suitable suggestions to the public.

\section{Conclusions}

Poor lifestyle habits including smoking history, irregular exercise and sedentary population are independent risk factors for getting the disease. Longer sleep time and taking moderate-intensity physical activity were protective factors against getting COVID-19. Sleep status and MET intensity classification are the influencing factors for length of hospitalization inpatients with COVID-19. Lack of sleep and low MET intensity may increase the risk of prolongedhospital stays. In the assessment of various physical activities, the impact of moderate physical activity MET is the most significant.In the global context of the COVID-19 outbreak, this encourages the public to carry out moderate physical activity and ensure adequate sleep to respond to the outbreak actively.

\section{Abbreviations}

COVID-19: Coronavirus disease 2019; SARS-CoV-2: Severe acute respiratory syndrome coronavirus 2; RTPCR: Real-time reverse-transcription polymerase-chain-reaction; CT: Computed tomography; IPAQ: International Physical Activity Questionnaire; NSF: National sleep foundation; OR: Odds ratio; Cl: Confidence interval; BMI: Body mass index; MET: Metabolic equivalent of task 


\section{Declarations}

\section{Ethics approval and consent to participate}

The ethical approval to use cartilages in this study was obtained from the Research and Ethics Committee of Chinese PLA General Hospital. All patients involved in this study signed informed consent orally.

\section{Consent for publication}

Not applicable

\section{Availability of data and material}

All data are presented in article or supplementary materials. If you need more data, please email to the corresponding author.

\section{Competing interests}

The authors declare that they have no conflict of interest.

\section{Funding}

This research is supported by the National Natural Science Foundation of China (No. 81901404)『Beijing

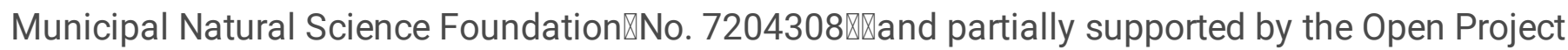
Program of the State Key Laboratory of Kidney Diseases in PLA General Hospital『No. KF2018-06区

\section{Authors' contributions}

Baozhen Huang*,Shixi Zhang* and Jiuyu Gong* contributes the same in this study. Pengtao Bao and Diangeng $\mathrm{Li}$ are the co-correspondence authors. Diangeng $\mathrm{Li}$ and Baozhen Huang designed the research study; Baozhen Huang conducted the research and wrote the manuscript; ,Shixi Zhang*,Jiuyu Gong*,Fengjuan Zhang, Lin Jiang,MingmingWang,JingCui,WeixinLi,Pengtao Bao collected the clinical data and conducted the questionnaire investigation. DiangengLi ,ChengJin and YanlinNiu revised the manuscript. All authors read and approved the final manuscript.

\section{Acknowledgments}

We declare the results of the study are presented clearly, honestly, and without fabrication, falsification, or inappropriate data manipulation, and the present study do not constitute endorsement by ACSM.

\section{References}


1.WHO main website. https://www.who.int (accessed March 31th, 2020).

2.Coronavirus disease(COVID-19) Situation Dashboard. https://www.who.int/redirect-pages/page/novelcoronavirus-(covid-19)-situation-dashboard(accessed 7 June 2020)

3.Wu Z, McGoogan J M. Characteristics of and important lessons from the coronavirus disease 2019 (COVID-19) outbreak in China: summary of a report of 72314 cases from the Chinese Center for Disease Control and Prevention[J]. Jama, 2020, 323(13): 1239-1242.

4.Zhou F, Yu T, Du R, et al. Clinical course and risk factors for mortality of adult inpatients with COVID-19 in Wuhan, China: a retrospective cohort study[J]. The lancet, 2020.

5. Yang J, Zheng Y, Gou X, et al. Prevalence of comorbidities in the novel Wuhan coronavirus (COVID-19) infection: a systematic review and meta-analysis[J]. International Journal of Infectious Diseases, 2020.

6.Cai H. Sex difference and smoking predisposition in patients with COVID-19[J]. The Lancet Respiratory Medicine, 2020, 8(4): e20.

7.Zhao Y, Zhao Z, Wang Y, et al. Single-cell RNA expression profiling of ACE2, the receptor of SARS-CoV2[Internet]. BioRxiv; 2020.Avaliable from:https://www.biorxiv.org/content/10.1101/2020.01.26.919985v2.full

8.Cai G. Bulk and single-cell transcriptomics identify tobacco-use disparity in lung gene expression of ACE2, the receptor of 2019-nCov [Internet]. medRxiv; 2020. Avaliable from:

https://www.medrxiv.org/content/medrxiv/early/2020/02/17/2020.02.05.20020107.full.pdf

9.Wu J T, Leung K, Bushman M, et al. Estimating clinical severity of COVID-19 from the transmission dynamics in Wuhan, China[J]. Nature Medicine, 2020: 1-5.

10.Vardavas C I, Nikitara K. COVID-19 and smoking: A systematic review of the evidence[J]. Tobacco induced diseases, 2020, 18.

11.Nishiura $\mathrm{H}$, Kobayashi T, Miyama T, et al. Estimation of the asymptomatic ratio of novel coronavirus infections (COVID-19)[J]. [Internet]. medRxiv; 2020. Avaliable

from:https://www.medrxiv.org/content/10.1101/2020.02.03.20020248v2

12.Corman V M, Landt O, Kaiser M, et al. Detection of 2019 novel coronavirus (2019-nCoV) by real-time RT-PCR[J]. Eurosurveillance, 2020, 25(3): 2000045.

13.Pianosi P T, Zhang Z, Hernandez P, et al. Measuring dyspnea and perceived exertion in healthy adults and with respiratory disease: new pictorial scales[J]. Sports medicine-open, 2016, 2(1): 17.

14.Diagnosis and Treatment Plan for COVID-19 (Trial Version 6). Chin Med J(Engl) 2020 Mar 17. 
15.Guan W, Liang W, Zhao Y, et al. Comorbidity and its impact on 1590 patients with Covid-19 in China: A Nationwide Analysis[J]. European Respiratory Journal, 2020.

16.0uyang $\mathrm{Y}$, Wang $\mathrm{H}$, Su $\mathrm{C}$, et al. Use of quantile regression to investigate changes in the body mass index distribution of Chinese adults aged 18-60 years: a longitudinal study. BMC Public Health. 2015;15:278. Published 2015 Mar 21. doi:10.1186/s12889-015-1606-8

17.US Department of Health and Human Services, and National Institutes of Health. Your guide to physical activity and your heart. No. 06-5714. NIH Publication, 2006.

18.Boon R M, Hamlin M J, Steel G D, et al. Validation of the New Zealand physical activity questionnaire (NZPAQ-LF) and the international physical activity questionnaire (IPAQ-LF) with accelerometry[J]. British journal of sports medicine, 2010, 44(10): 741-746.

19.IPAQ committee. Guidelines for data processing and analysis of the International Physical Activity Questionnaire (IPAQ). November 2005. Available from: http://www.ipaq.ki.se/scoring.html

20.Scholes S, Bridges S, Fat L N, et al. Comparison of the physical activity and sedentary behaviour assessment questionnaire and the short-form international physical activity questionnaire: an analysis of health survey for England data[J]. PloS one, 2016, 11(3).

21.National Sleep Foundation. National Sleep Foundation Recommends New Sleep Times. February 2, 2015).Available from https://www.sleepfoundation.org/press-release/national-sleep-foundationrecommends-new-sleep-times

22.Bai Y, Yao L, Wei T, et al. Presumed asymptomatic carrier transmission of COVID-19[J]. Jama, 2020, 323(14): 1406-1407.

23.Hu Z, Song C, Xu C, et al. Clinical characteristics of 24 asymptomatic infections with COVID-19 screened among close contacts in Nanjing, China[J]. Science China Life Sciences, 2020: 1-6.

24.Lai C C, Liu Y H, Wang C Y, et al. Asymptomatic carrier state, acute respiratory disease, and pneumonia due to severe acute respiratory syndrome coronavirus 2 (SARSCoV-2): Facts and myths[J]. Journal of Microbiology, Immunology and Infection, 2020.

25.Choi S M, Jeong Y J, Park J S, et al. The impact of lifestyle behaviors on the acquisition of pandemic (H1N1) influenza infection: a case-control study[J]. Yonsei medical journal, 2014, 55(2): 422-427.

26.Cohen S, Doyle W J, Alper C M, et al. Sleep habits and susceptibility to the common cold[J]. Archives of internal medicine, 2009, 169(1): 62-67.

27.Jung S, Akhmetzhanov A R, Hayashi K, et al. Real-time estimation of the risk of death from novel coronavirus (COVID-19) infection: inference using exported cases[J]. Journal of clinical medicine, 2020, 9(2): 523.

Page 12/16 
28. Haskell W L, Blair S N, Hill J O. Physical activity: health outcomes and importance for public health policy[J]. Preventive medicine, 2009, 49(4): 280-282.

29.Gautret P, Lagier J C, Parola P, et al. Hydroxychloroquine and azithromycin as a treatment of COVID-19: results of an open-label non-randomized clinical trial[J]. International journal of antimicrobial agents, 2020: 105949.

30.Cortegiani A, Ingoglia $\mathrm{G}$, Ippolito $\mathrm{M}$, et al. A systematic review on the efficacy and safety of chloroquine for the treatment of COVID-19[J]. Journal of critical care, 2020.

31.White $D$ B, Lo B. A framework for rationing ventilators and critical care beds during the COVID-19 pandemic[J]. Jama, 2020, 323(18): 1773-1774.

32.Manca M, Rusticali F, Gaddi A, et al. Physical activity, exercise and cardiovascular health[J]. Future Lipidology, 2006, 1(5): 513-516.

33.Kokkinos P. Physical activity, health benefits, and mortality risk[J]. ISRN cardiology, 2012, 2012.

34. Wilde B E, Sidman C L, Corbin C B. A 10,000-step count as a physical activity target for sedentary women[J]. Research quarterly for exercise and sport, 2001, 72(4): 411-414.

35. Hills A P, Andersen L B, Byrne N M. Physical activity and obesity in children[J]. British journal of sports medicine, 2011, 45(11): 866-870.

36.Tuan T C, Hsu T G, Fong M C, et al. Deleterious effects of short-term, high-intensity exercise on immune function: evidence from leucocyte mitochondrial alterations and apoptosis[J]. British journal of sports medicine, 2008, 42(1): 11-15.

37.Blond K, Brinkløv C F, Ried-Larsen M, et al. Association of high amounts of physical activity with mortality risk: a systematic review and meta-analysis[J]. British journal of sports medicine, 2019: bjsports-2018-100393.

\section{Tables}

Table 1. Results of multiple logistic regression on illness 


\begin{tabular}{|c|c|c|c|c|c|c|c|}
\hline Items & Categories & DF & Estimate & SE & $x^{2}$ & $P$ & OR $(95 \% \mathrm{Cl})$ \\
\hline Intercept & & 1 & 5.412 & 2.192 & 6.095 & 0.014 & \\
\hline $\begin{array}{l}\text { Smoke History (No } \\
\text { as reference区 }\end{array}$ & Yes & 1 & 1.093 & 0.376 & 8.447 & 0.004 & $\begin{array}{l}2.982(1.427- \\
6.231)\end{array}$ \\
\hline $\begin{array}{l}\text { Underlying Disease } \\
\text { (No as reference】 }\end{array}$ & Yes & 1 & 1.947 & 0.425 & 21.022 & $<0.001$ & $\begin{array}{l}7.008(3.049- \\
16.108)\end{array}$ \\
\hline $\begin{array}{l}\text { Regular Exercise } \\
\text { (Yes as reference) }\end{array}$ & No & 1 & 0.939 & 0.386 & 5.936 & 0.015 & $\begin{array}{l}2.558(1.202- \\
5.445)\end{array}$ \\
\hline $\begin{array}{l}\text { Sedentary } \\
\text { population (No as } \\
\text { reference) }\end{array}$ & Yes & 1 & 3.585 & 1.198 & 8.952 & 0.003 & $\begin{array}{l}\text { 36.054(3.444- } \\
377.443)\end{array}$ \\
\hline $\begin{array}{l}\text { Average daily sleep } \\
\text { time }(\mathrm{h})\end{array}$ & & 1 & -0.906 & 0.271 & 11.211 & 0.001 & $\begin{array}{l}0.404(0.238- \\
0.687)\end{array}$ \\
\hline \multirow{2}{*}{$\begin{array}{l}\text { Sleep Status } \\
\text { (Recommended as } \\
\text { reference) }\end{array}$} & $\begin{array}{l}\text { May Be } \\
\text { Appropriate }\end{array}$ & 1 & -2.421 & 0.524 & 21.315 & $<0.001$ & $\begin{array}{l}0.089(0.032- \\
0.248)\end{array}$ \\
\hline & Too Low & 1 & -2.097 & 0.894 & 5.501 & 0.019 & $\begin{array}{l}0.123(0.021- \\
0.709)\end{array}$ \\
\hline \multirow{2}{*}{$\begin{array}{l}\text { MET Intensity } \\
\text { Classification (High } \\
\text { as reference) }\end{array}$} & Moderate & 1 & -1.555 & 0.316 & 24.148 & $<0.001$ & $\begin{array}{l}0.211(0.114- \\
0.393)\end{array}$ \\
\hline & Low & 1 & -2.547 & 0.521 & 23.864 & $<0.001$ & $\begin{array}{l}0.078(0.028- \\
0.218)\end{array}$ \\
\hline
\end{tabular}

1. Significant level is 0.05 and significant $P$ values are shown in bold.

2. SE: Standard Error; OR: Odds Ratio; P: P value of multiple logistic regression

Table 2. Results of multiple logistic regression model on inpatient days 


\begin{tabular}{|c|c|c|c|c|c|c|}
\hline Variables & Categories & Estimate & SE & $x^{2}$ & $P$ & OR $(95 \% \mathrm{Cl})$ \\
\hline Intercept & Inpatient days $\geq 20$ & -1.802 & 0.291 & 38.316 & $<0.001$ & \\
\hline Intercept & $\begin{array}{l}\text { Inpatient days } \\
\text { between } 10 \text { and } 19\end{array}$ & 1.778 & 0.295 & 36.382 & $<0.001$ & \\
\hline \multirow[t]{2}{*}{$\begin{array}{l}\text { MET Intensity } \\
\text { Classification }\end{array}$} & Moderate & 0.594 & 0.365 & 2.656 & 0.103 & $\begin{array}{l}1.812(0.887- \\
3.701)\end{array}$ \\
\hline & Low & 1.898 & 0.725 & 6.864 & 0.009 & $\begin{array}{l}6.674(1.613- \\
27.613)\end{array}$ \\
\hline \multirow[t]{2}{*}{ Sleep Status } & Maybe Appropriate & 0.827 & 0.448 & 3.415 & 0.065 & $\begin{array}{l}2.287(0.951- \\
5.502)\end{array}$ \\
\hline & Lack of sleep & 1.709 & 0.745 & 5.271 & 0.022 & $\begin{array}{l}5.525(1.284- \\
23.770)\end{array}$ \\
\hline
\end{tabular}

1. SE: Standard Error; OR: odds ratio; P: $P$ value of $X^{2}$ test.

2. Score Test for the Proportional Odds Assumption: $X^{2}=0.3325, D F=2, P=0.8468$, which suggested that the model satisfies the assumption.

3. The reference for MET Intensity Classification and Sleep status are High and recommended respectively.

\section{Table 3. Results of Kruskal-Wallis $\mathrm{H}$ test in groups of hospital stay}




\begin{tabular}{|lllllll|}
\hline Variables & $\begin{array}{l}\text { Hospital stay } \\
\text { days }\end{array}$ & N & $\begin{array}{l}\text { Mean } \\
\text { Score }\end{array}$ & DF & H & $P$ \\
\hline Vigorous Activity MET*min & $0-9$ & 16 & 78.41 & 2 & 0.249 & 0.883 \\
\hline & $10-19$ & 108 & 82.60 & & & \\
\hline Moderate Activity MET*min & $\geq 20$ & 39 & 81.82 & & & \\
\hline & $0-9$ & 16 & 94.75 & 2 & 8.441 & $\mathbf{0 . 0 1 5}$ \\
\hline Walking MET*min & $10-19$ & 108 & 86.75 & & & \\
\hline & $\geq 20$ & 39 & 63.63 & & & \\
\hline & $0-9$ & 16 & 82.22 & 2 & 0.658 & 0.720 \\
\hline $\begin{array}{l}\text { Vigorous \& Moderate Activity } \\
\text { MET*min }\end{array}$ & $10-19$ & 108 & 83.27 & & & \\
\hline & 020 & 38 & 76.16 & & & \\
\hline & $10-19$ & 16 & 97.41 & 2 & 7.406 & 0.025 \\
\hline
\end{tabular}

Significant level is 0.05 and significant $\mathrm{P}$ values are shown in bold. DF is degrees of freedom.

\section{Supplementary Files}

This is a list of supplementary files associated with this preprint. Click to download.

- Supplementarymaterial1.docx

- SupplementaryTable6.docx

- SupplementaryTable5.docx

- SupplementaryTable4.docx

- SupplementaryTable3.docx

- SupplementaryTable2.docx

- Supplementarymaterial2.docx

- SupplementaryTable1.docx 